\title{
Stabile koronare Herzkrankheit - wann operieren?
}

\author{
Udo Sechtem, Ulrich Franke
}

Die medikamentöse Therapie hat bei stabiler KHK zentralen Stellenwert [1]. In den letzten Jahren wird die Frage nach einer zusätzlichen Revaskularisierung zunehmend diskutiert. Insbesondere in den USA ist die Interventionsrate bei symptomfreien oder oligosymptomatischen Patienten rückläufig. Welche Patienten mit stabiler Angina pectoris weiterhin von einer operativen Revaskularisierung profitieren, zeigt dieser Beitrag.

\begin{abstract}
Weniger Interventionen | Medikamentös wird die stabile KHK mit einer Kombination aus einem Cholesterinsenker (meist einem Statin) und einem Plättchenaggregationshemmer (meist $100 \mathrm{mg}$ Acetylsalicylsäure) behandelt [2]. Die Indikation zur Revaskularisierung wird in den letzten Jahren zunehmend kritisch hinterfragt - zumindest bei oligosymptomatischen Patienten. In den USA sind die Zahlen für Interventionen bei stabiler KHK seit Jahren rückläufig. Dies betrifft vor allem Bypassoperationen. In Massachusetts geht parallel auch die Rate an Perkutanen Koronarinterventionen (PCI) zurück [3]. In Deutschland hat sich dieser Trend bei der PCI noch nicht durchgesetzt.
\end{abstract}

Ursachen für den Rückgang I Für die zunehmend zurückhaltend gestellte Indikation spielen folgende Faktoren eine Rolle:

1. Die Häufigkeit von Myokardinfarkten hat insgesamt abgenommen - am ehesten aufgrund verbesserter primärer und sekundärer Präventionsmaßnahmen [4].

2. Im Publikationsjahr der COURAGE-Studie ist die Zahl der elektiven PCIs am stärksten gesunken. Die Studie hatte gezeigt, dass die PCI und die medikamentöse Therapie bezüglich harter Endpunkte (Myokardinfarkt, kardialer Tod) äquivalent sind [5].

3. In den USA wurde zudem genauer überprüft, ob revaskularisierende Maßnahmen angemessen sind. Dies hat den weiteren Rückgang begünstigt [6].

In der instabilen Situation ist die schnelle Revaskularisierung aber weiterhin unumstritten die Methode der Wahl.

\section{Gründe für eine Revaskularisierung}

Symptome beseitigen I Das vorrangige Ziel bei stabiler KHK ist es, die Symptomatik zu beseitigen. Die Koronarrevaskularisation ist diesbezüglich effektiv - und wirkt schneller als die medika- mentöse Therapie [7]. Insbesondere bei Patienten mit starken Angina-pectoris-Beschwerden ist die Revaskularisierung daher aus symptomatischen Gründen sinnvoll.

Prognose verbessern I Oft sind die thorakalen Missempfindungen nicht sehr ausgeprägt. In erster Linien verunsichern sie den Patienten in psychologischer Hinsicht: Sie erinnern daran, dass eine bedrohliche Herzerkrankung vorliegen kann. Bei diesen Patienten kann ein aufklärendes Gespräch über das meist niedrige Infarkt-und Todesrisiko dazu führen, dass die Beschwerden keine unangemessenen Ängste auslösen. Hier steht die Prognose im Vordergrund der Indikationsstellung.

Randomisierte Studien aus den 1980er Jahren haben gezeigt, dass eine Bypassoperation die Prognose bei oligosymptomatischen, stabilen Patienten mit angiografischer HochrisikoKonstellation verbessern kann [8-10].

Unter angiografischer Hochrisiko-Konstellation vesteht man eine

- $\geq 50 \%$-ige Stenose des linken Hauptstamms,

- koronare Dreigefäßerkrankung mit $\geq 70 \%$-igen proximalen Stenosen der drei koronaren Hauptäste oder $\geq 70 \%$-iger proximaler Stenose des Ramus interventricularis anterior (RIVA) und

- eine weitere $\geq 70 \%$-ige Stenose eines großen Koronarasts.

Es muss allerdings berücksichtigt werden, dass sich die medikamentöse Vergleichstherapie zum damaligen Zeitpunkt völlig von heutigen Standards unterscheidet.

\section{Revaskularisierung - ja oder nein?}

Kosten und Nutzen I Die Wahl der optimalen Therapieoption hängt vom individuellen NutzenRisiko-Verhältnis ab. Zu den Risiken einer Revaskularisierung gehören periprozeduraler Tod, 
Myokardinfarkt und Schlaganfall. Diese sind abzuwägen gegen die Vorteile, wie

- eine verbesserte Lebensqualität und

- eine verbesserte Prognose durch eine langfristig geringere Sterbe-, Myokardinfarkt- und Schlaganfallrate.

Für den ansonsten gesunden, aktiven Patienten ist das Behandlungsziel in aller Regel, die Brustschmerzen vollständig zu beseitigen und eine intensive physische Aktivität wieder zu ermöglichen.

Bei diesen Patienten hat die Revaskularisierung zunächst Vorteile, die aber im Vergleich mit der medikamentösen Therapie nach einem Jahr nicht mehr nachweisbar sind [7]. Für einen Teil der älteren Patienten mit ausgeprägter Angina pectoris und Hochrisikoprofil stehen häufig die heftigen Symptome im Vordergrund. Werden diese reduziert, sind die Tätigkeiten des täglichen Lebens wieder möglich - wenn auch verlangsamt.

Patienten mit niedrigem Risiko I Patienten mit geringem Risiko für die gefürchteten Ereignisse Myokardinfarkt oder Koronartod profitieren prognostisch nicht von einer Revaskularisierung [2]. Diese Patienten

- sind in der Regel gut belastbar,

- haben wenig oder keine objektiv nachweisbare Ischämie und

- haben angiografisch lediglich eine Ein- oder Zweigefäßerkrankung (oft mit distalen Stenosen) ohne Beteiligung des proximalen RIVA.

Abb. 1 Ereignisfreies Überleben ohne Tod, Myokardinfarkt oder Schlaganfall nach operativer Revaskularisierung bzw. medikamentöser Therapie. In der randomisierten BARI 2D-Studie [11] war die Überlebenswahrscheinlichkeit für Patienten nach Bypassoperation signifikant besser als bei alleiniger medikamentöser Behandlung. Einschlusskriterien waren Diabetes mellitus und KHK (angiografisch $\geq 50 \%$-ige Stenose einer größeren epikardialen Koronararterie und pathologischem Belastungstest bzw. $\geq 70 \%$-iger Stenose bei typischer Angina pectoris). Mit freundlicher Genehmigung [11].

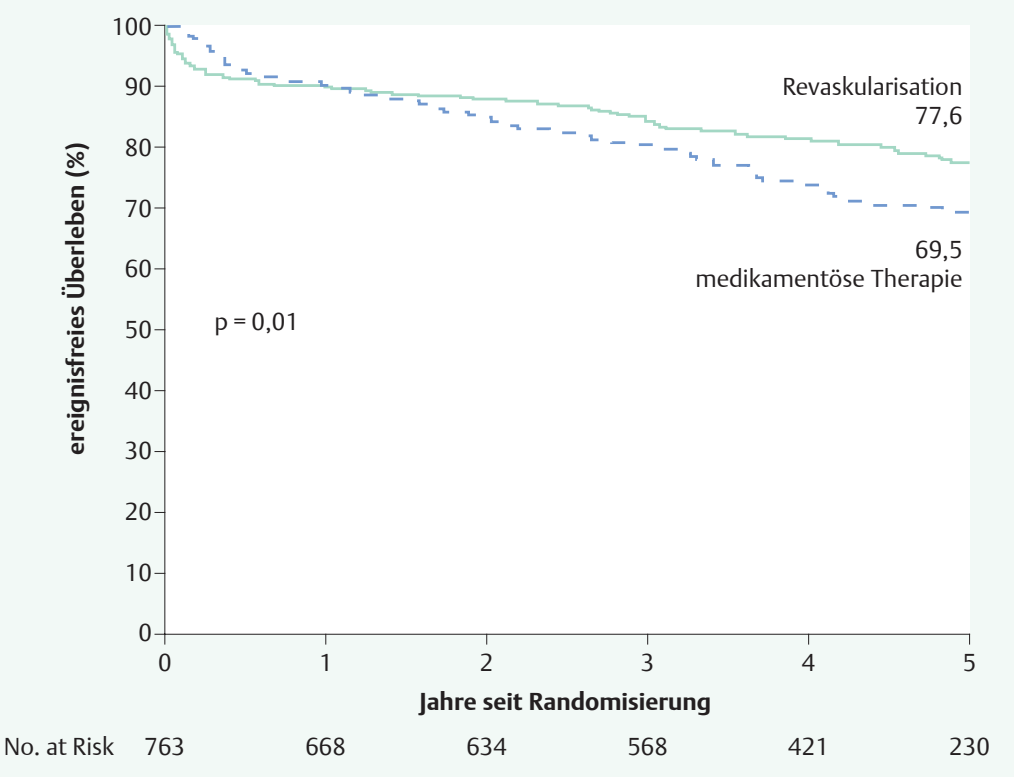

Patienten mit mittlerem Risiko | Bei ihnen ist

- die Belastbarkeit evtl. eingeschränkt,

- die Ischämie nicht sehr ausgeprägt (Bildgebung: < $10 \%$ des linksventrikulären Myokards) und

- es liegt angiografisch keine Hochrisiko-Konstellation vor.

Auch hier gibt es keine überzeugenden Daten die zeigen, dass die Revaskularisierung einen prognostischen Gewinn bringen kann - über eine Verbesserung der Symptomatik hinaus.

Patienten mit hohem Risiko I Hochrisikopatienten sind hingegen

- häufig wenig belastbar,

- haben eine stark beeinträchtigte Myokarddurchblutung ( $\geq 10 \%$ des linksventrikulären Myokards) und

- haben angiografisch eine Hochrisiko-Konstellation [2].

Bei diesen Patienten kann eine Bypassoperation die Prognose signifikant verbessern - auch im Vergleich zur heutigen medikamentösen Standardtherapie ( Abb. 1) [11].

Leitlinien-Empfehlungen I Die ESC-Leitlinien legen fest, in welchen Situationen eine Revaskularisierung aufgrund von prognostischen Vorteilen sinnvoll ist ( Tab. 1) [12]. Die US-Leitlinien fordern für die Indikation zur Revaskularisierung eine $>70 \%$-ige statt $>50 \%$-ige Stenose (außer bei Stenosen des linken Hauptstamms).

Studien haben gezeigt, dass Bypassoperationen die Prognose im Vergleich zur alleinigen medikamentösen Therapie verbessern können [15]. Für die PCl liegen keine Daten vor.

Voruntersuchungen | Gemäß den aktuellen ESCLeitlinien ( $\triangleright$ Tab. 1) muss vor der Revaskularisierung nachgewiesen werden, dass die Stenose-Konstellation auch wirklich die Ischämie verursacht. Dies kann entweder mit einem bildgebenden Verfahren oder durch die Messung der fraktionellen Flussreserve (FFR) erfolgen $[2,12]$. Ein pathologisches Belastungs-EKG erlaubt keine anatomische Zuordnung der Ischämie zu einem bestimmten Gefäß. Derartige Veränderungen können auch durch epikardiale Erkrankungen der Koronararterien ausgelöst werden, die häufig von einer mikrovaskulären Funktionsstörung begleitet werden [16].

\section{Bypass-Operation oder PCl?}

Vorteile der OP | Für die Koronarrevaskularisation kommen sowohl die perkutane interventionelle Therapie als auch die operative Bypassversorgung in Betracht. Der unbestreitbare Vorteil der Koronaroperation ist die exzellente Langzeitoffenheitsrate (abhängig vom verwendeten Graftma- 


\begin{tabular}{|c|c|c|c|}
\hline Therapieziel & Ausmaß der KHK (anatomisch bzw. funktionell) & Empfehlungsklasse & Empfehlungsgrad \\
\hline \multirow[t]{5}{*}{ Prognoseverbesserung } & Hauptstammstenose (>50\%) & I & $A$ \\
\hline & Jede proximale RIVA-Stenose > 50\% ${ }^{a}$ & 1 & A \\
\hline & $\begin{array}{l}\text { Zwei- oder Dreigefäßerkrankung mit Stenosen > 50\% } \\
\text { mit eingeschränkter LV-Funktion (LVEF }<40 \%)^{\mathrm{a}}\end{array}$ & I & A \\
\hline & Große Ischämiezone (> 10\% des LV) & 1 & B \\
\hline & $\begin{array}{l}\text { Letzte verbleibende offene Koronararterie mit } \\
\text { Stenose }>50 \%\end{array}$ & I & $\mathrm{C}$ \\
\hline Symptomverbesserung & $\begin{array}{l}\text { Jede Stenose }>50 \%{ }^{\text {a }} \text { mit einschränkender Angina oder } \\
\text { Angina-Äquivalent, wenn eine medikamentöse Therapie } \\
\text { keine Besserung bringt }\end{array}$ & I & $\mathrm{D}$ \\
\hline
\end{tabular}

Tab. 1 Indikationen zur Revaskularisation bei Patienten mit stabiler Angina pectoris oder stummer Ischämie (aus: Windecker S et al. 2014 ESC / EACTS Guidelines on Myocardial Revascularization. Eur Heart J 2014; 35: 2541-619 mit Genehmigung der ESC). KHK=Koronare Herzkrankheit, LV = linker Ventrikel, LVEF = linksventrikuläre Ejektionsfraktion, RIVA = Ramus interventricularis anterior

${ }^{a}$ mit dokumentierter regionaler Ischämie oder fraktioneller Flussreserve (FFR) $\leq 0,80$ bei Durchmesser-Stenosen $<90 \%$.

terial). Eine erneute Koronarbehandlung über die medikamentöse Therapie hinaus ist in den nächsten Jahren nur selten notwendig. Selbst im Falle eines Bypassverschlusses wird das Bypass-überbrückte, native Koronargefäß häufig noch eingeschränkt perfundiert.

Im Gegensatz zum koronaren Stentverschluss kommt es bei einem Bypassverschluss nicht zwangsläufig zu einem akuten Myokardinfarkt.

Diese Vorteile führen bei Patienten mit Dreigefäßerkrankung oder Hauptstammstenose bereits im ersten Jahr, stärker aber noch in den folgenden Jahren nach der Operation zu einer

- signifikant geringeren Myokardinfarktrate (3,8 vs. 9,7\% bei PCI; $\mathrm{p}<0,0001$ ) und

- signifikant geringeren erneuten Revaskularisierungswahrscheinlichkeit (13,7 vs. $25,9 \%$; $\mathrm{p}<0,0001$ ) [17]).

Die Gesamtsterblichkeit gegenüber PCI ist ebenfalls reduziert ( 11,4 vs. $13,9 \% ; p=0,10)$. Nachteil der Bypassoperation ist eine leicht erhöhte, prozedurbedingte zerebrale Insultrate (3,7 vs. $2,4 \%$; $\mathrm{p}=0,09)[17]$.

Komplexität entscheidet | Der Vorteil der Koronaroperation gegenüber der PCI wird bei Patienten mit Dreigefäßerkrankung oder Hauptstammstenose umso größer, je schwerer die koronare Herzkrankheit ist. Um die Komplexität der Erkrankung zu beschreiben, wurde der Syntax-Score etabliert. Dieser berücksichtigt sowohl die Anzahl als auch die Ausprägung der koronaren Verengungen.

- Methode der Wahl bei Patienten mit komplexer koronarer Herzerkrankung (Syntax-Score > 22) ist - bei vertretbarem operativem Risiko - die chirurgische Koronarrevaskularisation [12].

- Beide Revaskularisierungsstrategien kommen in Frage für Patienten mit
> koronarer Dreigefäßerkrankung oder Hauptstammstenose und einem Syntax-Score $\leq 22$ oder

- unkomplizierter Hauptstammstenose und einer Ein- bzw. Zweigefäßerkrankung mit proximaler RIVA-Stenose.

- Bei der koronaren Ein- und Zweigefäßerkrankung ohne proximale RIVA-Stenose liefert die PCI gleichwertige Ergebnisse [18]. Aufgrund der geringeren Morbidität bevorzugen die Leitlinien hier die PCI [12].

Kommen beide Therapieverfahren in Frage, sollten dem Patienten die Nachteile beider Verfahren dargestellt werden, $d$. h.

- die höhere Morbidität bei Bypass-OP und

- die höhere Reinterventionsrate bei PCI.

Komorbiditäten berücksichtigen | Komorbiditäten können das Risiko einer konventionellen Operation soweit erhöhen, dass andere Behandlungsstrategien die bessere Option sind, z. B.

- eine PCI,

- eine Kombination aus minimalinvasiver Operation und PCI (sogenannte Hybridoperation) oder

- die medikamentöse Therapie.

Patienten mit Diabetes mellitus haben in aller Regel eine diffusere und komplexere atherosklerotische Erkrankung der Koronargefäße. Entsprechend sind die Ergebnisse der operativen Revaskularisierung mittel- und langfristig besser als durch PCI [19]. Bei Patienten mit chronischer Niereninsuffizienz ist die Datenlage uneinheitlich. Mehrere Studien haben einen Überlebensvorteil nach Bypassoperation gezeigt, während es in anderen Studien keinen Unterschied zwischen Bypassoperation und PCI gab $[13,20]$.

Gemeinsam festlegen I Idealerweise sollte die weitere Therapie interdisziplinär in einem soge- 
nannten „Heart Team“ besprochen werden. Hierzu gehören konservative Kardiologen, KatheterInterventionalisten und Herzchirurgen. Bei der Therapiempfehlung berücksichtigt werden sollte

- die koronare Anatomie,

- die Symptomatik,

- das Lebensalter,

- die Komorbiditäten,

- die Wünsche des Patienten und

- die Erfahrung innerhalb des Krankenhauses und des einzelnen Operateurs.

Die technische Ausführung der koronaren Bypassoperation beeinflusst das Ergebnis signifikant. Die ESC-/ EACTS-Richtlinien enthalten hierzu erstmals detaillierte Empfehlungen [12].

Die Empfehlung des „Heart Teams“ muss dem Patienten in verständlicher Form vermittelt werden [13]. Für ein solches Gespräch muss ausreichend Zeit zur Verfügung stehen. Aus diesem Grund empfiehlt die europäische Leitlinie zur Myokardrevaskularisation die Diagnostik und Therapie zeitlich zu trennen.

Cave Die diagnostische Herzkatheteruntersuchung sollte nicht mit der Katheterintervention kombiniert werden [12].

$\mathrm{Zu}$ einer umfassenden Information gehört, dass der Patient über den zu erwartenden Verlauf und mögliche Komplikationen der verschiedenen Therapieverfahren aufgeklärt wird. Das Gespräch

Abb. 2 Bypass-Durchgängigkeitsraten über 15 Jahre aus retrospektiven Daten des Beth Israel Medical Center, New York (1851 konsekutive Patienten, von denen 278 symptomatische Patienten eine Koronarangiographie erhielten). LITA=linke Arteria thoracica interna, $R A=$ Arteria radialis, $S V(R A)=$ Venenbypass bei Patienten mit zusätzlichem Radialis-Bypass, SV(SV) = Venenbypass bei Patienten ohne zusätzlichem Radialis-Bypass. Mit freundlicher Genehmigung [27].

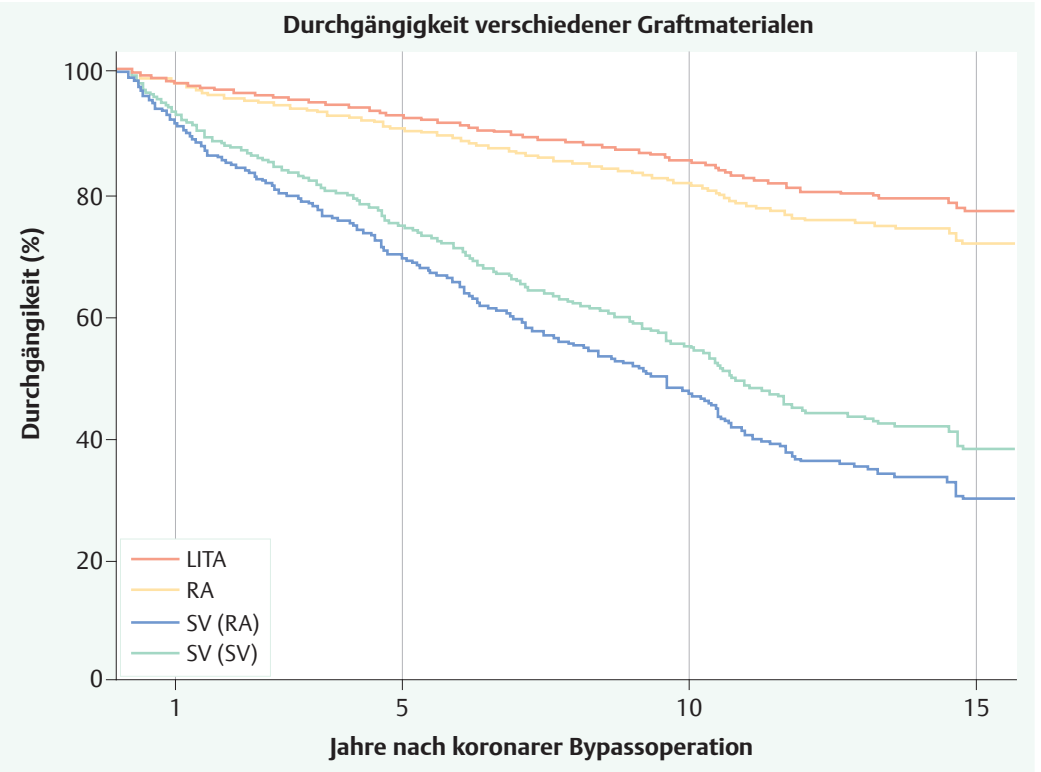

muss aber natürlich auch die individuellen Erwartungen und Wünsche des Patienten berücksichtigen.

\section{Welches Bypassmaterial?}

Autologes Material im Vorteil | Künstliche Bypassmaterialien haben nach wie vor keine akzeptablen Durchgängigkeitsraten. Deshalb wird für die koronare Bypasschirurgie nur autologes Graftmaterial verwendet. In Anbetracht der langen Überlebenszeiten nach einer Bypassoperation und der guten Ergebnisse von Zweit- und Drittoperationen muss dabei sparsam mit diesen autologen Gefäßen umgegangen werden.

Komplette Revaskularisierung | Die komplette Koronarrevaskularisation ist der inkompletten überlegen hinsichtlich Beschwerdefreiheit und Überleben [12, 21, 22]. Eine verbindliche Definition für die Vollständigkeit der Koronarrevaskularisation („completeness-of-revascularisation“) gibt es allerdings nicht. Basierend auf der chirurgischen Praxis versteht man im Allgemeinen unter kompletter Revaskularisierung die Bypassversorgung aller epikardialen Gefäße

- mit einem Durchmesser $>1,5 \mathrm{~mm}$ und

- mit einer Stenose von $>50 \%$ in mindestens einer angiografischen Ebene [23].

Nach einer Sternotomie sind die Koronargefäße gut erreichbar, weshalb hier die komplette Revaskularisierung angestrebt werden sollte.

Auswahl der Gefäße | Folgende Parameter der zu revaskularisierenden Koronararterien beeinflussen die Langzeitoffenheit der Bypässe:

- der Lumen-Durchmesser,

- der Stenosegrad proximal der Anastomose,

- das versorgte Koronarstromgebiet mit zugehörigem Myokard und

- manchmal die lokalen Gefäßwandverhältnisse im Anastomosenbereich.

Für die Revaskularisierung kommen autologe arterielle und venöse Gefäße in Frage. Das dominierende venöse Graft ist die V. saphena magna. Bei den Arterien kommen die beiden Aa. thoracicae internae (ITA; auch Aa. mammariae genannt) und Aa. radiales in Frage. Die V. saphena parva und die A. gastroepiploica werden nur noch selten in der Bypasschirurgie verwendet.

Brustwandarterien | Die Langzeitoffenheitsraten der ITA übertreffen die aller anderen Bypassgefäße deutlich: Nach 10 Jahren sind noch ca. 90\% der ITA-Grafts durchgängig. Dementsprechend wird heute für die Versorgung des RIVA der ITA-Bypass gefordert [7]. Werden beide Brustwandarterien verwendet, sinken die Risiken für Myokardinfarkt und eine erneute Koronarrevas- 
kularisation. Dadurch steigt die Überlebenswahrscheinlichkeit. Die positiven Effekte sind unabhängig vom Alter der Patienten und der linksventrikulären Funktion [24, 25]. Dies gilt auch für Diabetiker [26]. Die europäischen Leitlinien empfehlen deshalb, bei Patienten $<70$ Jahre beide ITA zu verwenden [7].

Die rechte und linke innere Brustwandarterie eignen sich am besten für die koronare Bypasschirurgie.

Werden beide Brustwandarterien genutzt, steigt allerdings das Risiko für tiefe sternale Wundinfektionen bzw. für Sternumdehiszenzen leicht an. Grund dafür ist die eingeschränkte Durchblutung des Brustbeins. Risikofaktoren für diese Komplikationen sind

- Alter,

- ausgeprägte Adipositas,

- schlecht eingestellter Diabetes mellitus und

- vorangegangene Bestrahlungen der Thoraxwand.

Die Datenlage hierzu ist allerdings widersprüchlich. Bei diesen Patienten sind Nutzen und Risiko der beidseitigen ITA-Verwendung abzuwägen. Verschiedene Methoden des Thoraxverschlusses und der Infektionsprophylaxe werden getestet, um die Inzidenz von tiefen Wundheilungsstörungen zu reduzieren.

Arteria radialis | Als weiteres arterielles Bypassmaterial kommt die A. radialis beider Arme als freies Graft in Frage. Die Offenheit in situ ist zwar schlechter als bei ITA ( Abb. 2), im Langzeitverlauf den Venengrafts aber überlegen [27]. Da diese Arterie in aller Regel eine starke, katecholaminsensible Lamina muscularis hat, neigt sie zur Spastik. Das ist für die Auswahl der koronaren Empfängergefäße wichtig. Diese müssen ausreichend groß sein und eine mindestens 70\%-ige vorgeschaltete Stenose aufweisen. Ansonsten kann es durch eine zu geringe Flussmenge durch die A. radialis zu einem Verschluss des Grafts kommen [28].

Vena saphena magna | Das in Deutschland immer noch am häufigsten verwendete Graftmaterial ist die V. saphena magna. Diese kann simultan zur Thorakotomie entnommen werden, was letztlich OP-Zeit spart. Ferner ist sie in großer Länge verfügbar, wodurch zahlreiche Einzelgrafts möglich sind. Die technisch anspruchsvolleren sequenziellen Anastomosen werden dadurch vermieden.

Bypässe mit zarten, schmalkalibrigen Venen können durchaus lange durchgängig bleiben. Dennoch sind nach 10 Jahren knapp die Hälfte - und nach 15 Jahren die überwiegende Anzahl - der Venenbypässe wieder verschlossen [27].
Endoskopische Graftentnahme I Die endoskopische Entnahme von A. radialis und V. saphena magna ist im Vergleich zur konventionellen offenen Technik deutlich schonender. Hierdurch können Wundheilungsstörungen drastisch minimiert werden. Allerdings ist das Verfahren deutlich teurer und wird in Deutschland nicht im DRG-System abgebildet.

\section{Mit oder ohne Herz-Lungenmaschine?}

Operationstechniken | Die Diskussion um die beste Strategie der koronaren Bypassoperation wird unter den Herzchirurgen sehr intensiv geführt. Der Vorteil eines Eingriffs mit Herz-Lungenmaschine (HLM) besteht darin, das Herz während der Bypassanlage ruhigzustellen. Bei der Operation am schlagenden Herzen kommt hingegen ein spezieller Haltearm zum Einsatz. Dieser saugt sich auf dem Bereich des Herzens fest, an dem die Koronaranastomose ausgeführt wird, und legt diesen still. Währenddessen muss der Rest des Herzens das notwendige Schlagvolumen generieren. Diese Technik wird OPCAB („off-pump-coronary-artery bypass“) genannt.

Vorteile der OPCAP | Bei der OPCAP-Methode muss die - nicht selten verkalkte - Aorta nicht kanüliert werden. Dadurch wird vermieden, dass sich Kalkpartikel lösen, die wiederum zu Schlaganfällen führen können. Vor allem aber hat das Blut des Patienten keinen Fremdoberflächenkontakt mit dem Schlauchsystem der HLM. Dieser kann das Entzündungssystems des Körpers aktivieren und ein SIRS („systemic-inflammatoryresponse-syndrome“) unterschiedlicher Schweregrade verursachen.

OP ohne Aortenkontakt I OPCAB erlaubt als einzige Technik eine Operation ohne Berührung der Aorta („Aortic-no-touch-Technik). Die linke ITA kann dabei als in-situ-Graft verwendet werden

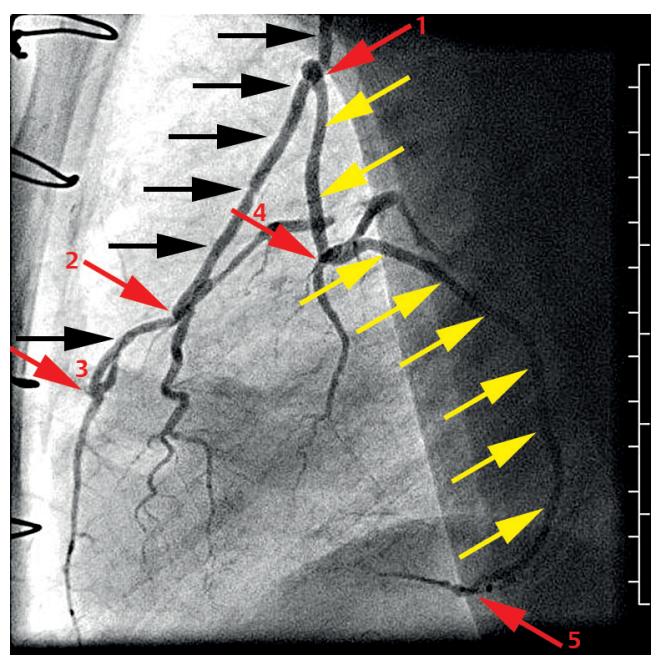

Abb. 3 Koronarangiogramm mit Kontrastmittelinjektion in die linke $A$. thoracica interna (LITA) nach vierfachem total-arteriellen Bypass in OPCAB-Technik. LITA (weiße Pfeile), Anastomosen (gelbe Pfeile), rechte $A$. thoracica interna (RITA, grüne Pfeile). End-zu-Seit-Anastomose von RITA an LITA (1), Seit-zu-SeitAnastomose von LITA an Ramus diagonalis (2), End-zu-SeitAnastomose LITA an Ramus interventricularis anterior (3), Seit-zu-Seit-Anastomose RITA an Ramus marginalis sinister (4). End-zu-Seit-Anastomose RITA an Ramus posterolateralis (5). 


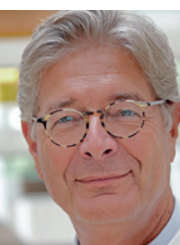

Prof. Dr. med. Udo Sechtem ist Chefarzt der Abteilung für Kardiologie, Robert-BoschKrankenhaus Stuttgart. udo.sechtem@rbk.de

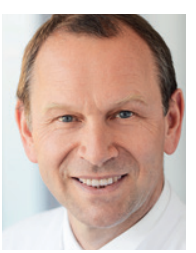

Prof. Dr. med.

Ulrich Franke

ist Chefarzt der Abteilung Herz- und Gefäßchirurgie, Robert-Bosch-Krankenhaus Stuttgart. ulrich.franke@rbk.de und wird weiterhin aus der A. subclavia gespeist. Das kann die Inzidenz neurologischer Komplikationen reduzieren. Um eine komplette Revaskularisierung zu ermöglich, kann die rechte ITA oder die A. radialis seitlich mit der linken ITA vereinigt werden ( $\bullet$ Abb. $\mathbf{3}$ ).

Studienlage I Drei der vier prospektiv randomisierten, multizentrischen Studien der letzten Jahre haben keine wesentlichen Unterschiede zwischen der konventionellen und der OPCABTechnik festgestellt. Der kombinierte Enpunkt bestand aus Tod, Myokardinfarkt, Apoplex und erneuter Revaskularisierung [29-32]. Die einzige Studie, die eine Überlegenheit der HLMOperationen nachgewiesen hat, war methodisch angreifbar [29]. Meta-Analysen und Datenbankauswertungen zufolge ist die Off-pump-Koronarrevaskularisation mit HLM hinsichtlich

- Mortalität,

- Schlaganfallrate,

- Nierenversagen und

- Nachblutung

tendenziell vorteilhafter [33, 34]. Die erneute Revaskularisierung ist hingegen nach On-pumpEingriffen ohne HLM etwas seltener.

Für einen erfolgreichen OPCAB-Eingriff scheint es entscheidend zu sein, die notfallmäßige Konversion zu einem HLM-Eingriff zu vermeiden [35]. Dafür sind die regelmäßige Durchführung und Erfahrung essenziell.

\section{Welcher Operations-Zugang?}

Sternotomie I Der dominierende Zugang für die operative Koronarrevaskularisation ist die komplette Sternotomie. Diese erlaubt es, alle betroffenen Koronararterien vollständig zu versorgen. $\mathrm{Zu}$ den postoperativen Komplikationen gehören tiefe Wundheilungsstörungen oder eine Pseudarthrose des Sternums. Dies kann mit anhaltenden Schmerzen oder einem deutlich verlängertem Krankenhausaufenthalt verbunden sein.

Seitliche Thorakotomie I Die Revaskularisierung über eine linksseitige, anterolaterale Thorakotomie ist eine attraktive Alternative zur Sternotomie. Etabliert ist dieses Verfahren für sogenannte MIDCAB-Eingriffe („minimally-invasive-coronary-artery-bypass“). Hierbei wird der RIVA minimalinvasiv mit der linken ITA revaskularisiert. Einige wenige Zentren gewinnen zunehmend mehr Erfahrung, auch Koronargefäße der Seitenund Hinterwand über diesen Zugang zu versorgen. Als zweites Bypass-Graft kommen dafür die A. radialis oder die V. saphena magna zum Einsatz, die dann seitlich an die linke ITA inseriert werden.

\section{Hybrideingriffe}

Die Vorteile vereinen | Koronare Hybrideingriffe kombinieren die minimalinvasive arterielle koronare Bypasschirurgie und die PCI. Das Verfahren wird angewandt, um eine speziellen Untergruppe von koronaren Mehrgefäßerkrankungen zu behandeln. Die Idee ist es, das Beste beider Methoden zu vereinen:

- die ausgezeichneten Durchgängigkeitsraten und Überlebensvorteile eines ITA-Graft zum RIVA und

- die guten Durchgängigkeitsraten von Medikamenten-freisetzenden Stents.

Letztere sind für Koronargefäße außer dem RIVA besser geeignet als Venenbypässe [36].

Studienlage I Das Kombinationsverfahren wurde Mitte der 1990er Jahre eingeführt. Seither gibt es nur eine kleine randomisierte Studie, die Hybrideingriffe mit der koronaren Bypasschirurgie vergleicht. In dieser Studie unterschieden sich die Ergebnisse zwischen den beiden Gruppen nicht [37]. Derzeit wird die Indikation für einen Hybrideingriff daher nur durch das „Heart Team“ gestellt [12].

\section{Konsequenz für Klinik und Praxis}

- Bei stabiler KHK ist primär die medikamentöse Behandlung die Therapie der Wahl.

- Eine zusätzliche Revaskularisierung kann erwogen werden, um die Symptome zu lindern und die Prognose zu verbessern.

- Insbesondere Hochrisikopatienten für Myokardinfarkt oder Koronartod können von der Revaskularisierung profitieren.

- Bei komplexer koronarer Anatomie verbessert die Bypassoperation die Prognose der Patienten im Vergleich zur PCl.

- Moderne Techniken haben die Invasivität des chirurgischen Eingriffs deutlich verringert.

\section{Literatur}

1 Iqbal J, Zhang Y], Holmes DR et al. Optimal medical therapy improves clinical outcomes in patients undergoing revascularization with percutaneous coronary intervention or coronary artery bypass grafting: insights from the Synergy Between Percutaneous Coronary Intervention with TAXUS and Cardiac Surgery (SYNTAX) trial at the 5-year follow-up. Circulation 2015; 131: 1269-1277

2 The Task Force on the management of stable coronary artery disease of the European Society of Cardiology. 2013 ESC guidelines on the management of stable coronary artery disease. Eur Heart ] 2013; 34: 2949-3003

3 Yeh RW, Mauri L, Wolf RE et al. Population trends in rates of coronary revascularization. JAMA Intern Med 2015; 175: 454-456

Vollständiges Literaturverzeichnis unter http://dx.doi.org/10.1055/s-0041-104814 
4 Ford ES, Ajani UA, Croft JB et al. Explaining the decrease in U.S. deaths from coronary disease, 1980-2000. N Engl J Med 2007; 356: 2388-2398

5 Boden WE, O‘Rourke RA, Teo KK et al. Optimal medical therapy with or without $\mathrm{PCl}$ for stable coronary disease. N Engl ] Med 2007; 356: 1503-1516

6 Patel MR, Dehmer G], Hirshfeld JW et al. ACCF / SCAI / STS / AATS / AHA / ASNC / HFSA / SCCT 2012 Appropriate use criteria for coronary revascularization focused update: a report of the American College of Cardiology Foundation Appropriate Use Criteria Task Force, Society for Cardiovascular Angiography and Interventions, Society of Thoracic Surgeons, American Association for Thoracic Surgery, American Heart Association, American Society of Nuclear Cardiology, and the Society of Cardiovascular Computed Tomography. J Am Coll Cardiol 2012; 59: 857-881

7 Weintraub WS, Spertus JA, Kolm P et al. Effect of $\mathrm{PCl}$ on quality of life in patients with stable coronary disease. N Engl J Med 2008; 359: 677-687

8 European Coronary Surgery Study Group. Long-term results of prospective randomised study of coronary artery bypass surgery in stable angina pectoris. Lancet 1982; 2: 1173-1180

9 CASS Principal Investigators and Their Associates. Myocardial infarction and mortality in the coronary artery surgery study (CASS) randomized trial. N Engl ] Med 1984; 310: 750-758

10 Detre KM, Takaro T, Hultgren $\mathrm{H}$ et al. Long-term mortality and morbidity results of the Veterans Administration randomized trial of coronary artery bypass surgery. Circulation 1985; 72: V84-89

11 Frye RL, August P, Brooks MM et al. A randomized trial of therapies for type 2 diabetes and coronary artery disease. N Engl J Med 2009; 360: 2503-2515

12 Windecker S, Kolh P, Alfonso F et al. 2014 ESC/EACTS Guidelines on myocardial revascularization: The Task Force on Myocardial Revascularization of the European Society of Cardiology (ESC) and the European Association for Cardio-Thoracic Surgery (EACTS) Developed with the special contribution of the European Association of Percutaneous Cardiovascular Interventions (EAPCI). Eur Heart J 2014; 35: 2541-2619

13 Fihn SD, Gardin JM, Abrams J et al. 2012 ACCF / AHA / ACP / AATS / PCNA/SCAI/STS Guideline for the diagnosis and management of patients with stable ischemic heart disease: a report of the American College of Cardiology Foundation / American Heart Association Task Force on Practice Guidelines, and the American College of Physicians, American Association for Thoracic Surgery, Preventive Cardiovascular Nurses Association, Society for Cardiovascular Angiography and Interventions, and Society of Thoracic Surgeons. J Am Coll Cardiol 2012; 60: e44-e164

14 Hillis LD, Smith PK, Anderson JL et al. 2011 ACCF / AHA Guideline for Coronary Artery Bypass Graft Surgery. A report of the American College of Cardiology Foundation / American Heart Association Task Force on Practice Guidelines. Developed in collaboration with the American Association for Thoracic Surgery, Society of Cardiovascular Anesthesiologists, and Society of Thoracic Surgeons. J Am Coll Cardiol 2011; 58: e123-e210
15 Sechtem U. Is FAME 2 a breakthrough for $\mathrm{PCl}$ in stable coronary disease? Clin Res Cardiol 2015; 104: 283-287

16 Ong P, Athanasiadis A, Hill S et al. Coronary microvascular dysfunction assessed by intracoronary acetylcholine provocation testing is a frequent cause of ischemia and angina in patients with exercise-induced electrocardiographic changes and unobstructed coronary arteries. Clin Cardiol 2014; 37: 462-467

17 Mohr FW, Morice MC, Kappetein AP et al. Coronary artery bypass graft surgery versus percutaneous coronary intervention in patients with three-vessel disease and left main coronary disease: 5-year follow-up of the randomised, clinical SYNTAX trial. Lancet 2013; 381: 629-638

18 Wu C, Camacho FT, Zhao S et al. Long-term mortality of coronary artery bypass graft surgery and stenting with drug-eluting stents. Ann Thorac Surg 2013; 95: 1297-1305

19 Farkouh ME, Domanski M, Sleeper LA et al. Strategies for multivessel revascularization in patients with diabetes. N Engl J Med 2012; 367: 2375-2384

20 Hage FG, Venkataraman R, Zoghbi G] et al. The scope of coronary heart disease in patients with chronic kidney disease. J Am Coll Cardiol 2009; 53 : 2129-2140

21 Rastan AJ, Walther T, Falk V et al. Does reasonable incomplete surgical revascularization affect early or long-term survival in patients with multivessel coronary artery disease receiving left internal mammary artery bypass to left anterior descending artery? Circulation 2009; 120: S70-77

22 Garcia S, Sandoval Y, Roukoz H et al. Outcomes after complete versus incomplete revascularization of patients with multivessel coronary artery disease: a meta-analysis of 89,883 patients enrolled in randomized clinical trials and observational studies. J Am Coll Cardiol 2013; 62: 1421-1431

23 Farooq V, Serruys PW, Garcia-Garcia HM et al. The negative impact of incomplete angiographic revascularization on clinical outcomes and its association with total occlusions: the SYNTAX (Synergy Between Percutaneous Coronary Intervention with Taxus and Cardiac Surgery) trial. J Am Coll Cardiol 2013; 61: 282-294

24 Kurlansky PA, Traad EA, Dorman M] et al. Thirty-year follow-up defines survival benefit for second internal mammary artery in propensitymatched groups. Ann Thorac Surg 2010; 90: 101-108

25 Lytle BW, Blackstone EH, Sabik JF et al. The effect of bilateral internal thoracic artery grafting on survival during 20 postoperative years. Ann Thorac Surg 2004; 78: 2005-2012

26 Dorman M], Kurlansky PA, Traad EA et al. Bilateral internal mammary artery grafting enhances survival in diabetic patients: a 30-year follow-up of propensity score-matched cohorts. Circulation 2012; 126: 2935-2942

27 Tranbaugh RF, Dimitrova KR, Friedmann P et al. Coronary artery bypass grafting using the radial artery: clinical outcomes, patency, and need for reintervention. Circulation 2012; 126: S170-175

28 Hayward PA, Gordon IR, Hare DL et al. Comparable patencies of the radial artery and right internal thoracic artery or saphenous vein beyond 5 years: results from the Radial Artery Patency and Clinical Outcomes trial. J Thorac Cardiovasc Surg 2010; 139: $60-65$ 
29 Shroyer AL, Grover FL, Hattler B et al. On-pump versus off-pump coronary-artery bypass surgery. N Engl J Med 2009; 361: 1827-1837

30 Lamy A, Devereaux PJ, Prabhakaran D et al. Off-pump or on-pump coronary-artery bypass grafting at 30 days. N Engl J Med 2012; 366: 1489-1497

31 Houlind K, Kjeldsen BJ, Madsen SN et al. On-pump versus off-pump coronary artery bypass surgery in elderly patients: results from the Danish on-pump versus off-pump randomization study. Circulation 2012; 125: 2431-2439

32 Diegeler A, Börgermann J, Kappert U et al. Off-pump versus on-pump coronary-artery bypass grafting in elderly patients. N Engl J Med 2013; 368: $1189-1198$

33 Kuss O, von Salviati B, Borgermann J. Off-pump versus on-pump coronary artery bypass grafting: a systematic review and meta-analysis of propensity score analyses. J Thorac Cardiovasc Surg 2010; 140: 829-835
34 Polomsky M, He X, O’Brien SM, Puskas JD. Outcomes of off-pump versus on-pump coronary artery bypass grafting: Impact of preoperative risk. J Thorac Cardiovasc Surg 2013; 145: 1193-1198

35 Chowdhury R, White D, Kilgo P et al. Risk factors for conversion to cardiopulmonary bypass during off-pump coronary artery bypass surgery. Ann Thorac Surg 2012; 93: 1936-1941

36 Panoulas VF, Colombo A, Margonato A et al. Hybrid coronary revascularization: promising, but yet to take off. J Am Coll Cardiol 2015; 65: 85-97

37 Gasior M, Zembala MO, Tajstra M et al. Hybrid revascularization for multivessel coronary artery disease. JACC Cardiovasc Interv 2014; 7: $1277-1283$ 